University of New Orleans

ScholarWorks@UNO

$1-10-2009$

\title{
Tilted parallel dielectric slab as a multilevel attenuator for incident p- or s-polarized light
}

\author{
R. M.A. Azzam \\ University of New Orleans, razzam@uno.edu
}

Follow this and additional works at: https://scholarworks.uno.edu/ee_facpubs

Part of the Electrical and Electronics Commons, and the Optics Commons

\section{Recommended Citation}

R. M. A. Azzam, "Tilted parallel dielectric slab as a multilevel attenuator for incident p- or s-polarized light," Appl. Opt. 48, 425-428 (2009).

This Article is brought to you for free and open access by the Department of Electrical Engineering at ScholarWorks@UNO. It has been accepted for inclusion in Electrical Engineering Faculty Publications by an authorized administrator of ScholarWorks@UNO. For more information, please contact scholarworks@uno.edu. 


\title{
Tilted parallel dielectric slab as a multilevel attenuator for incident $p$ - or $s$-polarized light
}

\author{
R. M. A. Azzam \\ Department of Electrical Engineering, University of New Orleans, \\ New Orleans, Louisiana 70148, USA (razzam@uno.edu) \\ Received 30 October 2008; revised 16 December 2008; accepted 16 December 2008; \\ posted 18 December 2008 (Doc. ID 103418); published 9 January 2009
}

\begin{abstract}
Under the condition of first-order blooming, a parallel dielectric slab, which is inserted in the path of an obliquely incident $p$ - or $s$-polarized light beam, introduces multiple discrete attenuation levels given by $1 / 3,4 / 27,4 / 243, \ldots \ldots$ in reflection and $4 / 9,4 / 81,4 / 729, \ldots \ldots$ in transmission. These attenuation levels are independent of the slab refractive index, incident $p$ or $s$ linear polarization, or the presence of identical transparent surface coatings at the front and back sides of the slab. Therefore, the tilted slab provides multidecade reflectance and attenuation reference values that can be used in calibrating spectrophotometers and filters, and also for testing the linearity of photodetectors. For an uncoated dielectric slab, incidence angles that cause first-order blooming are determined as functions of the slab refractive index for incident $p$ - or $s$-polarized light. (C) 2009 Optical Society of America

OCIS codes: $\quad 120.1840,120.5240,230.0230,260.0260$.
\end{abstract}

\section{Introduction}

Optical attenuators that reduce the intensity of a light beam by a predetermined amount are useful for precise spectrophotometry [1], calibrating filters [2], testing the linearity of photodetectors [3-5], and in applications that involve high-power lasers [6-10]. Existing designs include the rotating-sector attenuator [1], a three-polarizer system that consists of two fixed outer polarizers and a rotating middle polarizer [2], variable-gap optical-tunneling (frustrated-total-internal-refection) attenuator [6,7], wedged-plate attenuator [8], and reflective mültimirror systems at oblique incidence [10].

In this paper an optically isotropic dielectric parallel slab (of zero-wedge angle) is shown to function as a multilevel attenuator for obliquely incident $p$ - or $s$-polarized light, when the slab is oriented to achieve maximum first-order reflection. First-order blooming leads to a set of discrete attenuation levels that depend only on the number of internal reflections inside the slab but that are independent of the slab refractive index, incident ( $p$ or $s$ ) linear polarization,

0003-6935/09/020425-04\$15.00/0

(C) 2009 Optical Society of America or the presence of identical transparent surface layers at the front and back sides of the slab. Tilted dielectric slabs are also key elements of divisionof-amplitude photopolarimeters that are used for partial or complete measurement of the state of polarization of light [11-13].

In Section 2 the principle of operation of the multilevel parallē-dielectric-slab attenuator under the first-order blooming condition is considered. The analysis of Section 2 is independent of the selected $p$ or $s$ linear polarization, slab refractive index, or the presence of identical surface layers at the front and back of the slab. In Sections 3 and 4 uncoateddielectric-slab attenuators for incident $s$ and $p$ linearly polarized light are considered separately, and the corresponding incidence angles that lead to first-order blooming are obtained as functions of the slab refractive index. Section 5 gives a brief summary of the results presented in this paper.

\section{Principle of Operation}

Figure 1 shows the reflection and transmission (in air) of a light beam by a homogeneous and optically isotropic dielectric slab of refractive index $n$ and uniform thickness $d$ at an angle of incidence $\phi$. (The slab thickness $d$ is chosen sufficiently large compared to 


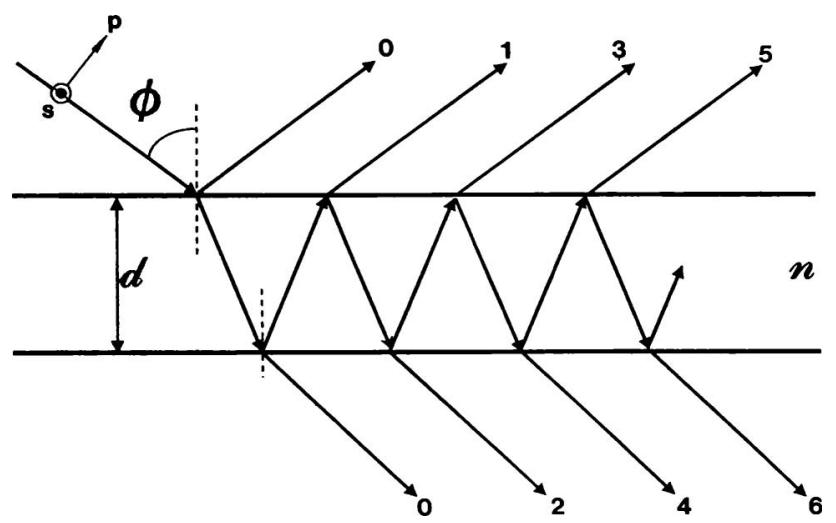

Fig. 1. Multiple reflection and transmission (in air) of a light beam by a homogeneous optically isotropic dielectric slab of refractive index $n$ and uniform thickness $d$ at an angle of incidence $\phi . p$ and $s$ represent the linear polarizations parallel and perpendicular to the plane of incidence, respectively.

the beam diameter to prevent overlapping of the parallel beams that exit the slab from either side.) If unit-intensity $p$ - or $s$-polarized incident light is assumed, the intensity $I_{m}$ of the $p$ - or $s$-polarized beam that exits the slab after $m$ internal reflections is given by

$$
I_{m}=R_{\nu}{ }^{m}\left(1-R_{\nu}\right)^{2}, \nu=p, s, \quad m \geq 1 .
$$

In Eq. (1) $R_{\nu}$ is the zeroth-order intensity reflectance of the air-slab interface for the $\nu$ polarization. Odd order numbers $(m=1,3,5, \ldots)$ correspond to the reflected beams and even order numbers $(m=2,4,6, \ldots)$ represent the transmitted ones. Substitution of $m=0$ in Eq. (1) gives the intensity of the zeroth-order transmitted beam.

Figure 2 shows the intensity $I_{m}$ of the $m$ th-order beam plotted as a function of front-surface reflectance $R_{\nu}$ for different values of $m$. It is apparent that $I_{m}$ reaches a maximum at a given value of $R_{\nu}$ that depends on the order number $m$. The condition of blooming of the $m$ th order is determined by setting the derivative of $I_{m}$ with respect to $R_{\nu}$ equal to zero:

$$
\partial I_{m} / \partial R_{\nu}=R_{\nu}{ }^{m-1}\left(1-R_{\nu}\right)\left[m-(m+2) R_{\nu}\right]=0 .
$$

Equation (2) has only one acceptable solution:

$$
R_{\nu}=m /(m+2) .
$$

The corresponding maximum intensity of the $m$ thorder beam is obtained from Eqs. (1) and (ㅁ) as

$$
I_{m}(\max )=\frac{4 m^{m}}{(m+2)^{m+2}}
$$

For example, 1st- and 2nd-order blooming occurs when $R_{\nu}=1 / 3$ and $R_{\nu}=1 / 2$. The corresponding intensity maxima of these two orders are $I_{1}(\max )=$ $4 / 27=0.148148 \quad$ and $\quad I_{2}(\max )=1 / 16=0.0625$, respectively. Figure 2 readily confirms these results. Blooming of any order $m$ is attained when the

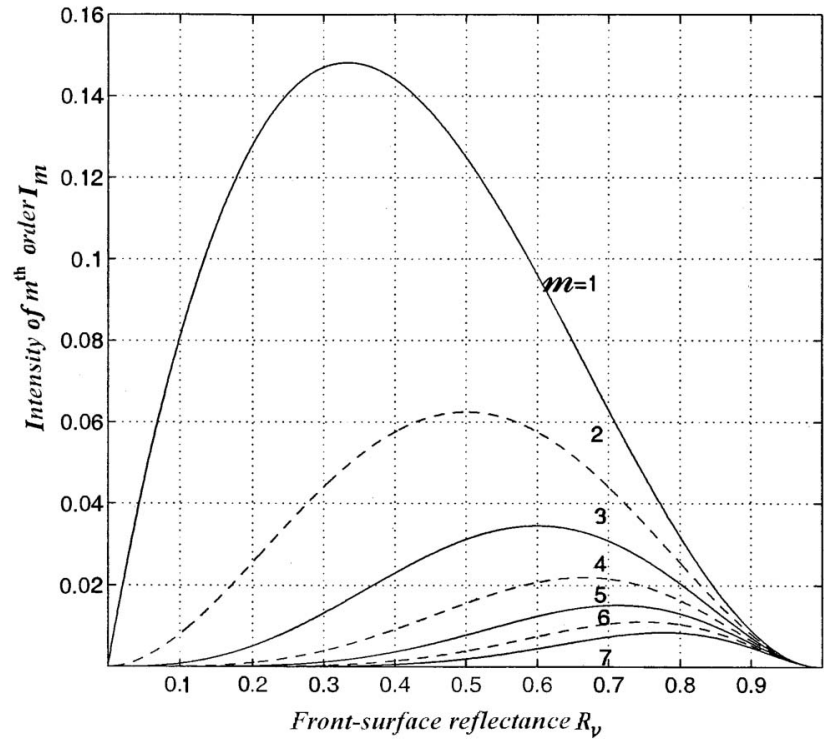

Fig. 2. Intensity $I_{m}$ of the $m$ th-order beam that exits the slab of Fig. 1 plotted as a function of the front-surface reflectance $R_{\nu}$ for different values of the order number $m$. $(\nu=p, s$ represent the linear polarizations parallel and perpendicular to the plane of incidence, respectively.) Continuous and dashed lines correspond to the reflected and transmitted orders, respectively.

front-surface reflectance $R_{\nu}$ is adjusted (by tilting the slab) to take the specific value given by Eq. (3).

For the attenuator application 1st-order blooming is of particular interest. Under this condition, the slab (which is inserted in the path of incident $p$ - or $s$-polarized light beam) introduces the following discrete attenuation levels: $1 / 3,4 / 27,4 / 243, \ldots \ldots$. in reflection and $4 / 9,4 / 81,4 / 729, \ldots \ldots$ in transmission. Again, it is important to emphasize that these discrete attenuation levels are the same regardless of the slab refractive index $n$, incident $p$ or $s$ linear polarization, or the presence of identical transparent surface coatings at the front and back sides of the slab.

Another interesting consequence of first-order blooming is that the sum of intensities of all reflected orders is the same as the sum of intensities of all transmitted orders and equals $1 / 2$ when the slab is presumed lossless.

\section{Uncoated-Parallel-Slab Attenuator for Incident s-Polarized Light}

The Fresnel amplitude reflection coefficient for $s$-polarized light which is incident from air on an uncoated dielectric slab of refractive index $n$ at an angle of incidence $\phi$ is given by

$$
r_{s}=\frac{\cos \phi-\left(n^{2}-\sin ^{2} \phi\right)^{1 / 2}}{\cos \phi+\left(n^{2}-\sin ^{2} \phi\right)^{1 / 2}} .
$$

Equation (ㅁ) can be solved for $n^{2}$ :

$$
n^{2}=\sin ^{2} \phi+\left(1-\sin ^{2} \phi\right)\left(\frac{1-r_{s}}{1+r_{s}}\right)^{2} .
$$


First-order blooming occurs when $R_{s}=1 / 3$, hence $r_{s}=-1 / \sqrt{3}$, where the minus sign is consistent with the Nebraska-Muller conventions [14,15]. Substitution of $r_{s}=-1 / \sqrt{3}$ in Eq. (6) and solving for $\sin ^{2} \phi, \phi=\phi_{s}$, we obtain

$$
\sin ^{2} \phi_{s}=\left(\frac{(7+4 \sqrt{3})-n^{2}}{(6+4 \sqrt{3})}\right) .
$$

Equation (7) indicates that the slab refractive index $n$ must be limited to the range

$$
n \leq 2+\sqrt{3} .
$$

Most optical materials that are transparent in the visible and IR spectrum have refractive indices [16] that satisfy Eq. (8). For example, for a glass slab with $n=1.5$, Eq. (5) predicts first-order blooming at $\phi_{s}=78.772^{\circ}$. This result was confirmed experimentally by reflecting an $s$-polarized, $633 \mathrm{~nm}$, He-Ne laser beam by a $10 \mathrm{~mm}$ thick polished optical glass parallel plate and rotating the slab until first-order blooming is achieved. The incidence angle measured by a precision $\left(0.005^{\circ}\right)$ goniometer agreed with the theoretical result to within $0.1^{\circ}$. Further improvement in the precise determination of the angular position of maximum first-order reflection can be achieved by superimposing a small (e.g., $0.5^{\circ}$ ) torsional oscillation and using a lock-in amplifier to zero the first harmonic of the detector signal at the modulation frequency.

\section{Uncoated-Parallel-Slab Attenuator for Incident p-Polarized Light}

For incident $p$-polarized light, first-order blooming occurs below the Brewster angle $\left(\phi_{B}=\tan ^{-1} n\right.$ when the amplitude reflectance at the air-slab interface $r_{p}=+1 / \sqrt{3}$. By substituting $r_{p}=+1 / \sqrt{3}$ in the Fresnel amplitude reflection coefficient for the $p$ polarization,

$$
r_{p}=\frac{n^{2} \cos \phi-\left(n^{2}-\sin ^{2} \phi\right)^{1 / 2}}{n^{2} \cos \phi+\left(n^{2}-\sin ^{2} \phi\right)^{1 / 2}},
$$

the incidence angle $\phi=\phi_{p}$ of first-order blooming is obtained,

$$
\sin ^{2} \phi_{p}=\left(\frac{n^{2}\left[n^{2}-(7+4 \sqrt{3})\right]}{\left[n^{4}-(7+4 \sqrt{3})\right]}\right), \quad n \geq 2+\sqrt{3} .
$$

For an uncoated Ge slab $(n=4)$, Eq. (7) gives $\phi_{p}=21.719^{\circ}$. Because of its weak dispersion, a multireflection Ge slab is a suitable candidate as an attenuator for incident $p$-polarized light over a broad IR spectral range [16].

First-order blooming of $p$-polarized light can also take place above the Brewster angle when $r_{p}=-1 / \sqrt{3}$. (Note that the plus and minus signs, or the 0 or $\pi$ reflection phase shifts, are consistent with the Nebraska-Muller conventions [14,15].) By substitution of $r_{p}=-1 / \sqrt{3}$ in Eq. (9), we obtain the corresponding angle of incidence, $\phi=\phi_{p}$, of firstorder blooming:

$$
\sin ^{2} \phi_{p}=\left(\frac{n^{2}\left[n^{2}-(7-4 \sqrt{3})\right]}{\left[n^{4}-(7-4 \sqrt{3})\right]}\right), \quad n \leq 2+\sqrt{3} .
$$

For a glass slab with $n=1.5$, one obtains $\phi_{p}=$ $82.293^{\circ}$ from Eq. (11). Based on Eq. (11) the minimum incidence angle for first-order blooming of $p$-polarized light above the Brewster angle is $\phi_{p}=82.229^{\circ}$, which corresponds to $n=1.401226$. Such high angles are obviously unsuitable and render $p$-polarization attenuators that use low-index slabs impractical.

If both sides of a low-index (e.g., glass) slab are coated with a quarter-wave-thick high-index (e.g., $\mathrm{TiO}_{2}$ ) film, first-order blooming of $p$ - or $s$ polarized light becomes possible at lower angles of incidence [17].

\section{Summary}

The use of a tilted parallel dielectric slab as a multilevel attenuator for incident $p$ - or $s$-polarized light has been described. Multidecade discrete attenuation levels are attained under the condition of first-order blooming. These levels are independent of the slab refractive index, incident $p$ or $s$ linear polarization, or the presence of identical transparent surface coatings at the front and back sides of the slab. Potential applications of these simple attenuators include calibration of spectrophotometers and filters and testing the linearity of photodetectors. For use of the device as a possible attenuation or reflectance standard the effect of residual absorption, surface roughness, surface flatness, small wedge angle, temperature, stress-induced birefringence, and spectral bandwidth should be considered.

\section{References}

1. J. E. Stewart, "A rotating-sector attenuator of adjustable transmittance for precise spectrophotometry," Appl. Opt. 1, 75-77 (1962).

2. H. E. Bennett, "Accurate method for determining photometric linearity," Appl. Opt. 5, 1265-1270 (1966).

3. C. L. Sanders, "A photocell linearity tester," Appl. Opt. 1, 207-211 (1962)

4. W. Budde, "Multidecade linearity measurements on $\mathrm{Si}$ photodiodes," Appl. Opt. 18, 1555-1558 (1979).

5. W. Budde, "Large-flux-ratio linearity measurements on $\mathrm{Si}$ photodiodes," Appl. Opt. 21, 3699-3701 (1982).

6. T. Oseki and S. Saito, "A precision variable, double prism attenuator for $\mathrm{CO}_{2}$ lasers," Appl. Opt. 10, 144-149 (1971).

7. K. Wilner and N. P. Murarka, "Design considerations and test results of an evanescent switch-attenuator," Appl. Opt. 20, 3600-3604 (1981).

8. K. Bennett and R. L. Byer, "Computer-controllable wedgedplate variable attenuator,” Appl. Opt. 19, 2408-2412 (1980). 
9. H. Lotem, A. Eyal, and R. Shuker, "Variable attenuator for intense unpolarized laser beams," Opt. Lett. 16, 690-692 (1991).

10. J. H. Lehman, D. Livigni, X. Li, C. L. Cromer, and M. L. Dowell, "Reflective attenuator for highenergy laser measurements," Appl. Opt. 47, 3360-3363 (2008).

11. A. M. El-Saba, R. M. A. Azzam, and M. A. G. Abushagur, "Parallel-slab division-of-amplitude photopolarimeter," Opt. Lett. 21, 1709-1711 (1996).

12. R. M. A Azzam, A. M. El-Saba, and M. A. G. Abushagur, "Spectrophotopolarimeter based on multiple reflections in a coated dielectric slab," Thin Solid Films 313/314, 53-57 (1998).
13. R. M. A. Azzam, "Parallel-slab polarizing beam splitter and photopolarimeter," Appl. Opt. 46, 292-294 (2007).

14. R. H. Muller, "Definitions and conventions in ellipsometry," Surf. Sci. 16, 14-33 (1969).

15. R. M. A. Azzam and N. M. Bashara, Ellipsometry and Polarized Light, (North-Holland, 1987).

16. W. J. Tropf, M. E. Thomas, and T. J. Harris, "Properties of crystals and glasses," in Handbook of Optics, Vol. II, M. Bass, E. W. Van Stryland, D. R. Williams, and W. L. Wolfe, eds. (McGraw-Hill, 1995), Chap. 33.

17. R. M. A. Azzam and F. F. Sudradjat, "Reflection of $p$ - and $s$-polarized light by a quarter-wave layer: explicit expressions and applications to beam splitters," Appl. Opt. 47, 11031108 (2008). 